\title{
Diez años de experiencia de la Academia Mexicana de Cirugía en la promoción de las próximas generaciones de investigadores
}

\author{
Ten years of experience of the Mexican Academy of Surgery in the promotion of the next \\ generations of researchers
}

\author{
Jorge Loría-Castellanos ${ }^{1,2,3 *}$, Felipe Cruz-Vega',2, Emilio Arch-Tirado ${ }^{2,4}$, Álvaro J. Montiel-Jarquín ${ }^{3,5}$, \\ Erandy Montes de Oca-García ${ }^{6}$ y Juan C. Sánchez-Echeverría ${ }^{1}$
}

${ }^{1}$ División de Proyectos Especiales en Salud, Instituto Mexicano del Seguro Social (IMSS), Ciudad de México; ${ }^{2}$ Academia Mexicana de Cirugía, Ciudad de México; ${ }^{3}$ Academia Nacional de Educación Médica, Ciudad de México; ${ }^{4}$ Centro Neurológico, Hospital ABC Santa Fe, Ciudad de México; Unidad Médica de Alta Especialidad, Ciudad de México; ${ }^{5} \mathrm{Hospital}$ de Especialidades Puebla, Instituto Mexicano del Seguro Social (IMSS), Puebla; ${ }^{6}$ Hospital General 197, Instituto Mexicano del Seguro Social (IMSS), Estado de México. México

\section{Resumen}

Objetivo: Describir los primeros 10 años del Concurso Interuniversitario y de Médicos Residentes de la Academia Mexicana de Cirugía. Método: Estudio observacional de tipo transversal descriptivo en el que se consideran las variables categoría de participación (universitario/residente), tipo de trabajo (investigación, caso clínico, etc.), universidad e institución de procedencia, y tipo de presentación (cartel/oral). Se empleó estadística descriptiva. Resultados: Desde 2010 se han desarrollado nueve concursos interuniversitarios y ocho de médicos residentes. En total se han presentado 974 trabajos, predominando los de residentes (65.29\%), las presentaciones orales (54.51\%) y los trabajos originales (58.11\%). El 8.9\% de los trabajos han sido publicados. Conclusiones: El Concurso es un buen foro para difundir la investigación de las nuevas generaciones de médicos.

Palabras Clave: Productividad científica. Estudiantes. Residentes.

\begin{abstract}
Objective: To describe the productivity of the research works presented in the Interuniversity and resident doctors Competition of the Mexican Academy of Surgery since its origin in 2010. Method: Observational cross-sectional descriptive study in which the variables are considered: category of participation (university/resident) type of work (research, clinical case, etc.), university and institution of origin, type of presentation [poster/oral]) Descriptive statistics was used. Results: Since 2010, 9 interuniversity competitions and 8 resident doctors have been developed. A total of 974 papers were presented, predominantly residents (65.29\%), oral presentations (54.51\%) and original works (58.11\%). 8.9\% of the published works. Conclusions: The Contest is a good forum to disseminate research in the new generations of doctors.
\end{abstract}

Key Words: Scientific productivity. Students. Residents.

\footnotetext{
Correspondencia:

*Jorge Loría-Castellanos

Paseo de la Reforma, 476

Col. Juárez, Del. Cuauhtemoc

Fecha de recepción: 01-08-2019

C.P. 06600, Ciudad de México, México

E-mail: jloriac@ hotmail.com

Fecha de aceptación: 08-10-2019

DOI: $10.24875 / C I R U .19001461$

0009-7411/@ 2019 Academia Mexicana de Cirugía. Publicado por Permanyer. Este es un artículo open access bajo la licencia CC BY-NC-ND (http://creativecommons.org/licenses/by-nc-nd/4.0/).
} 


\section{Introducción}

Al desarrollar investigación científica el alumno no solo adquiere conocimientos, sino que se ubica ante la inmejorable oportunidad de, a través del método científico, poder desarrollar habilidades, actitudes y valores que le ayuden a solucionar problemas con independencia y creatividad; todo esto de vital importancia dentro de la formación médica ${ }^{1,2}$.

El éxito de la investigación estudiantil radica en una buena preparación de los docentes y en una adecuada promoción y acompañamiento a los estudiantes con procesos de tutoría efectiva ${ }^{3}$.

A pesar de lo anterior, se han reconocido limitaciones para desarrollar de forma efectiva esta actividad en el área médica, situación que se profundiza en Latinoamérica, lo que impacta de manera negativa en las próximas generaciones.

Constituida en 1933, la Academia Mexicana de Cirugía es una corporación científica asociación civil integrada por médicos cirujanos y profesionistas afines, dedicada a la investigación, el estudio, el fomento, el perfeccionamiento y el desarrollo educativo de las disciplinas médico-quirúrgicas. Analiza, plantea y realiza acciones en beneficio de la salud de la población de los Estados Unidos Mexicanos, y mantiene relaciones e intercambio académico con personas físicas y morales nacionales e internacionales ${ }^{4}$.

Siendo congruentes con esta inquietud, la Academia Mexicana de Cirugía se propuso establecer dentro de su mayor evento anual, la Semana Quirúrgica Nacional, una nueva forma de fomentar la investigación, el Concurso Interuniversitario y de Médicos Residentes, un foro para que tanto los médicos en formación como los próximos especialistas compartan sus experiencias en materia de investigación. La primera edición de este concurso se dio en el año 2010 dentro de la 52. ${ }^{a}$ Semana Quirúrgica Nacional desarrollada en la Ciudad de Puebla, en la que solo se incluyeron presentaciones en cartel de participantes universitarios. Al paso de los años, y en vista del interés y de la inquietud de los participantes, el Concurso se abrió a los residentes de las diferentes especialidades, creándose en el afán de ser más inclusivos diferentes modalidades de presentación: presentaciones orales o en cartel electrónico, trabajos de investigación original, casos clínicos y revisiones.

Este trabajo presenta los principales resultados en la productividad del Concurso Interuniversitario y de Médicos Residentes en sus primeros 9 años.

\section{Método}

Estudio observacional de tipo transversal analítico en el que se analizaron las características de los trabajos de investigación presentados en el Concurso Interuniversitario y de Médicos Residentes, desde su inicio en 2010 hasta la convocatoria de 2018. Se empleó estadística descriptiva.

\section{Resultados}

Desde su origen en 2010, ha habido nueve ediciones del Concurso Interuniversitario y ocho del de Médicos Residentes (Tabla 1). De forma inicial solo participaron trabajos universitarios (54) en cartel, y para el año 2011 se consideró la inclusión de médicos residentes de posgrado de las diferentes especialidades. A partir de 2013, las presentaciones de cada categoría se dividieron en orales o cartel electrónico, en tanto que las evaluaciones de los trabajos se realizaron según los siguientes grupos: investigación original, casos clínicos y revisiones.

Han sido 974 trabajos los que se han presentado desde 2010 en este Concurso, de los cuales el $65.29 \%$ (636) fueron realizados por residentes. Han predominado las presentaciones orales (54.51\%) y los trabajos originales (58.11\%).

Un total de 3273 autores-coautores han colaborado en los trabajos presentados, pertenecientes a 21 Estados de la República, 31 universidades y 54 instituciones asistenciales. De la categoría de universitarios, 127 de los participantes han vuelto a presentar trabajos en convocatorias posteriores en las categorías de residentes; de la categoría de residentes, 189 participantes han participado como coautores-asesores una vez concluida su especialidad.

Se ha observado un incrementado paulatino en el número de trabajos al paso de los años: de 54 en el año 2010 a 192 en el año 2018.

Predominan las presentaciones con temas de cirugía, con un $44.76 \%$, seguidas de medicina interna (24.02\%) y Gineco-obstetricia (10.36\%) (Tabla 2).

En ambas categorías predominan las presentaciones de casos clínicos y los estudios de prevalencia, aunque llama la atención que es mayor el número de trabajos experimentales en la categoría de universitarios (Tabla 3). El porcentaje de trabajos originales también se ha incrementado con el tiempo, siendo del $37.03 \%$ en el primer concurso y del $67.18 \%$ en el más reciente. Desde sus inicios, 87 (8.9\%) de los trabajos han sido publicados. 


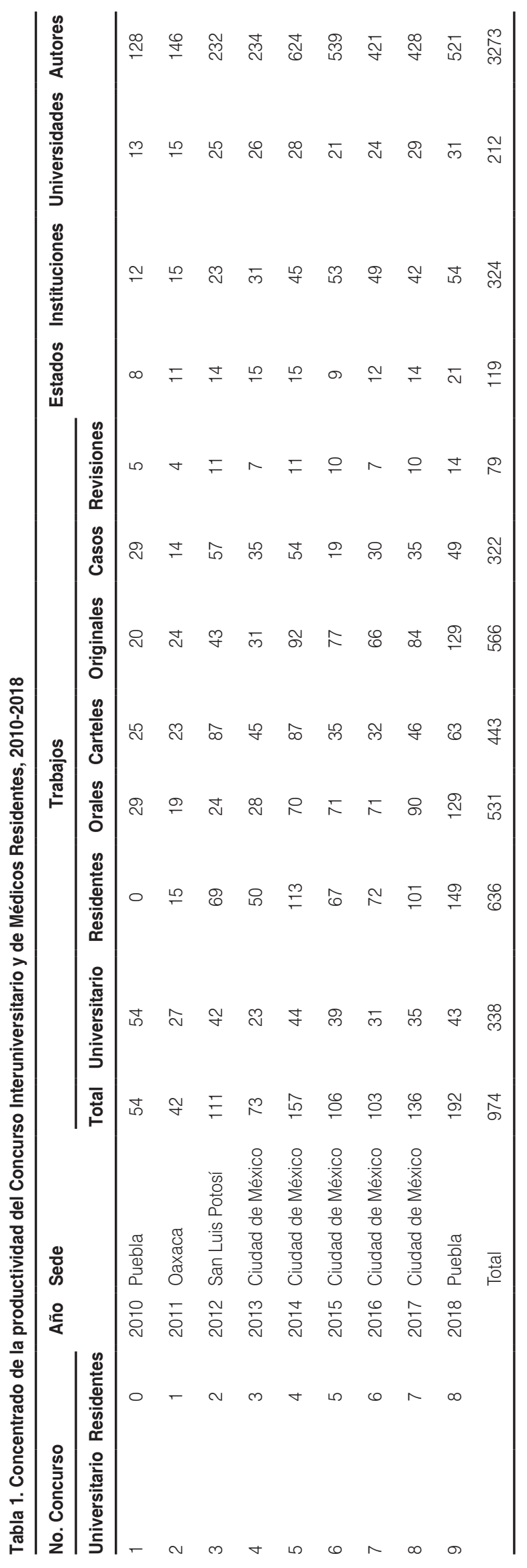

Tabla 2. Distribución por especialidades de los 974 trabajos presentados en el Concurso Interuniversitario y de Médicos Residentes, 2010-2018

\begin{tabular}{lc}
\hline Cirugía & 436 \\
Medicina interna & 234 \\
Gineco-obstetricia & 101 \\
Pediatría & 76 \\
Gastroenterología & 45 \\
Oftalmología & 30 \\
Terapia intensiva & 21 \\
Urgencias & 12 \\
Misceláneos & 10 \\
Historia de la medicina & 5 \\
Medicina aeroespacial & 4 \\
Total & 974 \\
\hline
\end{tabular}

\section{Discusión}

Entendiendo la ciencia como un fenómeno social, la actividad científica está íntimamente relacionada con otras prácticas de la sociedad 5 .

Un punto clave de la formación de los profesionales de la salud es conocer e implementar de forma adecuada el método científico, aterrizado en las situaciones reales a las que se enfrenta día a día.

Es una realidad que se considera que la investigación, tanto en estudiantes como en residentes, es pobre; esto por muchas causas, entre las que podrían mencionarse la falta de interés de los estudiantes, la falta de una verdadera cultura en investigación por parte de los tutores, o la falsa idea de que la investigación es solo para los grupos «de elite»"-8.

En este sentido, tanto el desarrollo de proyectos de investigación como su difusión resultan piedras angulares que trascienden la calidad de vida de los pacientes e impactan realmente en los sistemas de salud 6 .

Los estudios realizados en Latinoamérica reportan que, a pesar de que los estudiantes de ciencias de la salud están motivados para realizar investigaciones, son pocos los que llegan a presentar sus trabajos en congresos, considerando que entre las principales limitantes se encuentran la falta de espacios propicios, la falta de asesoría y el considerar que sus trabajos no cuentan con la calidad necesaria ${ }^{7,8}$.

Es de llamar la atención el constante crecimiento en el número de participantes, de 54 en la primera edición a 192 en la más reciente, lo que sin duda nos 
Tabla 3. Distribución por tipo de estudio de los 974 trabajos presentados en el Concurso Interuniversitario y de Médicos Residentes, 2010-2018

\begin{tabular}{lccccccccc}
\hline & $\begin{array}{c}\text { Caso } \\
\text { clínico }\end{array}$ & Prevalencia & $\begin{array}{c}\text { Serie de } \\
\text { casos }\end{array}$ & $\begin{array}{c}\text { Casos y } \\
\text { controles }\end{array}$ & Cohortes & $\begin{array}{c}\text { Cuasi } \\
\text { - experimental }\end{array}$ & Experimental & $\begin{array}{c}\text { Revisión } \\
\text { sistemática }\end{array}$ & $\begin{array}{c}\text { Otros } \\
\text { Universitarios }\end{array}$ \\
\hline 190 & 99 & 19 & 16 & 10 & 6 & 56 & 7 & 19 \\
Residentes & 201 & 106 & 50 & 44 & 56 & 26 & 35 & 9 & 25 \\
Total & 391 & 205 & 69 & 60 & 66 & 32 & 91 & 16 & 44 \\
\hline
\end{tabular}

habla tanto de una mejor difusión del evento como de un mayor interés de los participantes.

Otro dato que cabe señalar es la inversión que ha tenido el tipo de trabajos presentados, que de forma inicial se inclinaban hacia las revisiones y los casos clínicos, y en los últimos concursos se han orientado hacia investigaciones originales. En esto seguramente influye el mayor número de participantes de las residencias médicas, que utilizan este foro para presentar los avances de sus tesis de especialidad.

Era esperable que, dada la esencia de la Academia Mexicana de Cirugía, predominaran los temas quirúrgicos en las presentaciones, pero es de destacar el incremento en la presencia de temas "clínicos", resaltando también la inclusión de temas «novedosos», como serían los de medicina aeroespacial. En esto seguramente influye la apertura que ha tenido la convocatoria, así como el proceso de difusión entre las diferentes universidades, instituciones y sedes de las especialidades de todo el país.

Aunque predominaron los estudios considerados más «sencillos» (casos clínicos y estudios de prevalencia), un número interesante de universitarios presentaron trabajos experimentales. En esto muy probablemente influye su inclusión desde los ciclos básicos en grupos de investigación de sus propias universidades.

Más que una mera reunión académica, el Concurso se ha constituido como un foro en donde los participantes, al compartir sus experiencias, permiten tanto generar líneas de estudio como fomentar el interés por la investigación. Es trascendental el número de participantes que al paso del tiempo no solo continúan participando, sino que ahora guían y asesoran a las nuevas generaciones. Es interesante que los participantes de las primeras ediciones del concurso se han transformado en tutores de las últimas convocatorias, promoviendo la participación de sus alumnos, lo que refleja un impacto real en las nuevas y próximas generaciones de investigadores.

Aunque la frecuencia de publicación de los trabajos es similar a la de otras series reportadas ${ }^{9}$, queda pendiente la tarea de trabajar en generar una mayor y mejor cultura de publicación en la formación académica de los estudiantes de pregrado y posgrado.

\section{Conclusiones}

- La modalidad del Concurso se constituye como un foro multidisciplinario para compartir experiencias en los diferentes niveles y áreas de la medicina.

- Se ha incrementado la participación dentro del Concurso Interuniversitario y de Médicos residentes.

- Es necesario desarrollar una verdadera cultura de la investigación, tanto en los alumnos como en los tutores.

\section{Conflicto de intereses}

Los autores confirman no tener ningún conflicto de intereses ni haber recibido financiamiento.

\section{Responsabilidades éticas}

Protección de personas y animales. Los autores declaran que los procedimientos seguidos se conformaron a las normas éticas del comité de experimentación humana responsable y de acuerdo con la Asociación Médica Mundial y la Declaración de Helsinki.

Confidencialidad de los datos. Los autores declaran que han seguido los protocolos de su centro de trabajo sobre la publicación de datos de pacientes.

Derecho a la privacidad y consentimiento informado. Los autores han obtenido el consentimiento informado de los pacientes y/o sujetos referidos en el artículo. Este documento obra en poder del autor de correspondencia.

\section{Bibliografía}

1. Bascó Fuentes EL, Barbón Pérez OG, Solís Cartas U, Poalasín Narváez LA, Pailiacho Yucta H. Diagnóstico de la actividad científica estudiantil en la carrera de Medicina de la Universidad Nacional de Chimborazo. Educ Med. 2017;18:154-159. 
2. Toro-Huamanchumo CJ, Failoc-Rojas VE, Díaz-Vélez C. Participación en sociedades científicas estudiantiles y en cursos extracurriculares de investigación, asociados a la producción científica de estudiantes de medicina humana: estudio preliminar. Educ Med. 2015;18:293-8.

3. Mejia Álvarez CR, Quiñones Laveriano DM, Chacón Mostacero J, Aguirre Valenzuela EA, Miñan Tapia VAL, Rosas Varillas AM. Médicos investigadores: percepción de estudiantes de medicina y factores asociados a la posibilidad de serlo. Educación Médica Superior. 2017; 31:53-63.

4. Academia Mexicana de Cirugía. Acerca de la AMC. (Consultado el 6 de septiembre de 2018.) Disponible en: http://amc.org.mx/historia.php

5. Corrales-Reyes IE, Rodríguez García MJ, Reyes Pérez JJ, García Raga M. Limitantes de la producción científica estudiantil. Educ Med. 2017;18:199-202
6. González RMR, Sánchez SY, Alcaide GY, Vázquez VMJ, Arteaga VB, Cece GL. Comportamiento de las publicaciones científicas. Revista Cubana de Educación Médica Superior. 2015;29(3).

7. Corrales-Reyes IE, Rodríguez García MJ, Reyes Pérez JJ, García Raga M. Limitantes de la producción científica estudiantil. Educ Med. 2017;18:199-202.

8. Toro-Polo M, Pereyra-Elías R, Nizama-Vía A, Ng-Sueng LF, VélezSegovia E, Galán-Rodas E, et al. Publicación de los trabajos presentados a los congresos cientíicos de estudiantes de medicina, Perú 2002-2009: características y factores asociados. Rev Peru Med Exp Salud Publica. 2012;29:461-8.

9. Valladares-Garrido MJ, Flores-PérezI, Failoc-Rojas VE, Marinas-Miranda W, Valladares-Garrido D, Mejía CR. Publicación de trabajos presentados a congresos científicos internacionales de estudiantes de medicina de Latinoamérica, 2011-2014. Educ Med. 2017;18:167-73. 